\title{
Distinguishing between intramural pregnancy and choriocarcinoma: A case report
}

\author{
SHAN SU ${ }^{1,2^{*}}$, DEVENDRA CHAVAN ${ }^{1 *}, \mathrm{KUN} \mathrm{SONG}^{1}$, DENNIS CHI ${ }^{3}$, \\ GUIYU ZHANG ${ }^{1}$, XIAOHUI DENG ${ }^{1,2}, \mathrm{LI} \mathrm{LI}^{1,2}$ and BEIHUA KONG ${ }^{1}$ \\ ${ }^{1}$ Department of Obstetrics and Gynecology; ${ }^{2}$ Reproductive Medicine Center, Qilu Hospital Affiliated to Shandong University, \\ Jinan, Shandong 250012, P.R. China; ${ }^{3}$ Gynecology Service, \\ Department of Surgery, Memorial Sloan Kettering Cancer Center, New York, NY 10021, USA
}

Received October 13, 2015; Accepted December 6, 2016

DOI: $10.3892 / \mathrm{ol} .2017 .5737$

\begin{abstract}
Intramural pregnancy is a rare type of ectopic pregnancy with an unclear etiology. It may be associated with uterine wall injury and/or abnormal uterine conditions, such as adenomyosis, in certain cases. In the present report, a case of intramural pregnancy associated with adenomyosis is discussed. The patient was 34 years old and presented with amenorrhea for 40 days. Ultrasonography and magnetic resonance imaging revealed a mixed echogenic mass located within the posterior wall of the uterine fundus with abundant blood flow. In addition, the patient's $\beta$-human chorionic gonadotropin levels were markedly elevated; however, these levels demonstrated a declining tendency. Clinically, it was difficult to distinguish the diagnosis of the case between intramural pregnancy and choriocarcinoma. Following initial treatment with methotrexate-based chemotherapy, a laparotomy was performed to confirm the diagnosis and excise the lesion. Pathological analysis confirmed a diagnosis of intramural pregnancy and adenomyosis within the uterine wall. The results of the present case report suggest that surgical intervention should be the first action performed when intramural pregnancy is suspected, in order to confirm the diagnosis and treat the disease.
\end{abstract}

\section{Introduction}

Intramural ectopic pregnancy refers to a pregnancy located within the uterine wall, without a connection to the fallopian tubes or endometrial cavity. Intramural pregnancy is a rare

Correspondence to: $\mathrm{Dr} \mathrm{Li} \mathrm{Li}$, Department of Obstetrics and Gynecology, Qilu Hospital Affiliated to Shandong University, 107 Wenhuaxi Road, Jinan, Shandong 250012, P.R. China

E-mail: bzlily@163.com

*Contributed equally

Key words: intramural ectopic pregnancy, choriocarcinoma, adenomyosis condition that constitutes $<1 \%$ of ectopic pregnancies (1). The etiology of intramural pregnancy is unclear. A previous medical history of uterine surgical procedures, such as cesarean section, myomectomy, curettage or hysteroscopy, are considered to be risk factors for intramural pregnancy (2). Other pre-disposing factors include the following: A history of ectopic pregnancy and tubal surgery; smoking; in vitro fertilization and embryo transfer (IVF-ET); endometriosis; diethylstilbestrol exposure; and intrauterine device placement (3). The clinical symptoms of intramural pregnancy are nonspecific, as patients may present with no obvious symptoms, mild vaginal bleeding and abdominal pain or, conversely, hypovolemic shock due to a uterine rupture.

Choriocarcinoma is a highly malignant epithelial tumor caused by trophoblastic proliferation, which may be secondary to any type of pregnancy. While the uterus is frequently affected, choriocarcinoma may metastasize to other organs, such as the lung, vagina, liver and brain. It is difficult to diagnosis intramural pregnancies at an early stage due to their varied manifestations, which can mimic trophoblastic disorders, uterine fibroids and other uterine wall conditions. Life-threatening complications, such as uterine ruptures, can develop if intramural pregnancies are not diagnosed and treated at an early stage.

\section{Case report}

A 34-year-old woman, gravida 2 para 1, was referred to the Department of Obstetrics and Gynecology at Qilu Hospital Affiliated to Shandong University with suspected gestational trophoblastic disease (GTD) in April 2015. The patient's first pregnancy was uncomplicated there was no past history of miscarriage or termination. Menstrual history was unremarkable and the last menstrual period had occurred 40 days prior to admission. The physical examination revealed no abnormalities. An ultrasonography (USG) scan performed upon admission revealed a mixed echogenic mass measuring $5.1 \times 4.6 \mathrm{~cm}$ located within the posterior wall of the uterine fundus with an abundant blood supply. The mass was separate from the uterine cavity (Fig. 1). The patient's serum $\beta$-human chorionic gonadotropin ( $\beta$-hCG) level was $14,153 \mathrm{mIU} / \mathrm{ml}$. On the second day of admission, the patient's serum $\beta$-hCG level 

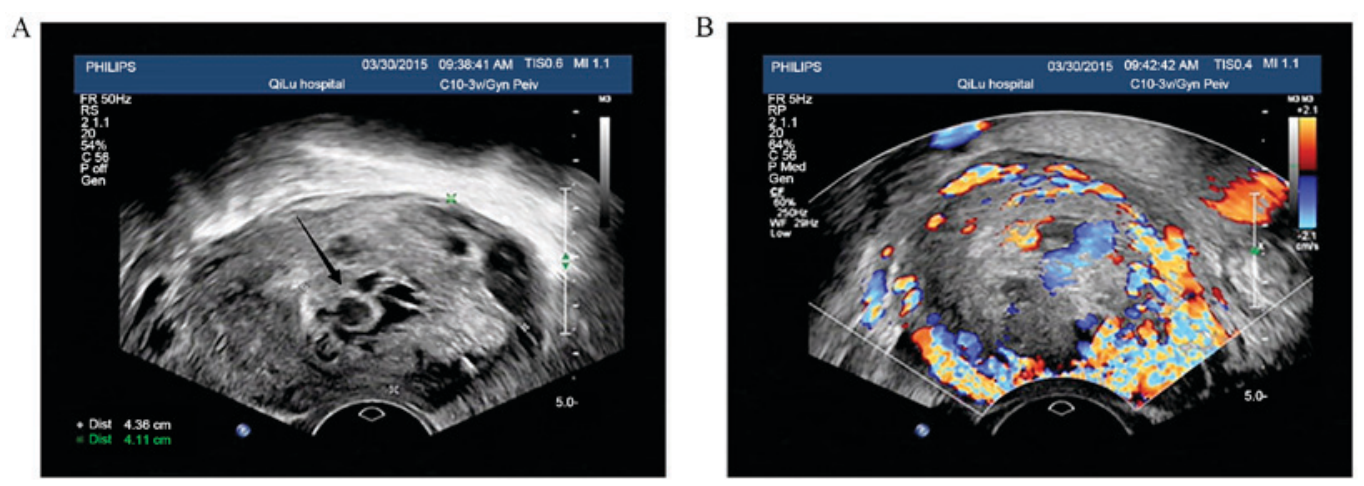

Figure 1. Ultrasonographic images of the lesion. (A) Transvaginal ultrasonography revealed a solid cystic lesion completely confined to the myometrium of left uterine wall with no connection to the endometrial cavity. The black arrow indicates the gestation sac. (B) Doppler images revealed abundant perilesional vascularity.

A

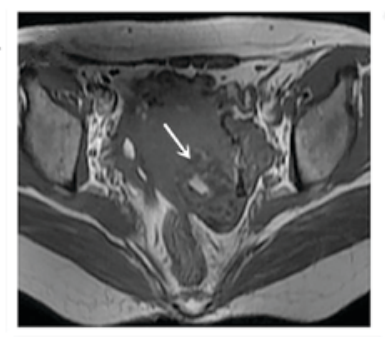

C

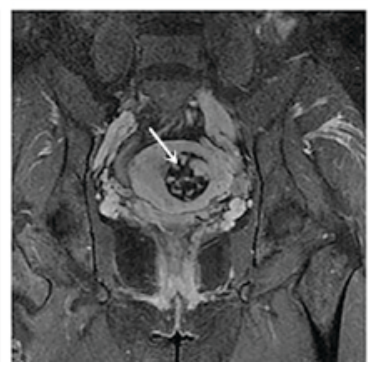

B

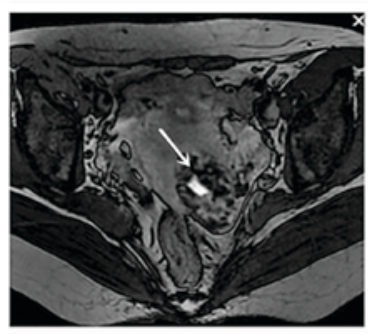

D

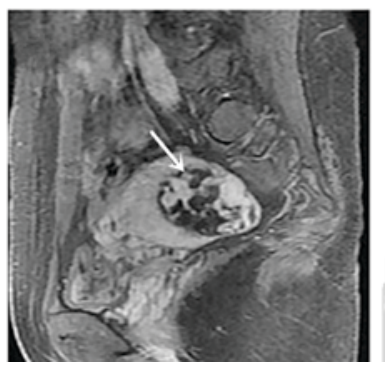

Figure 2. MRI images of the lesion. MRI imaging revealed a mass with (A) low signal intensity on T1WI and (B) high signal intensity on T2WI. (C) T2WI, sagittal plane. (D) T2WI, coronal plane. Arrows indicate the lesion. T1WI, T1-weighted image; T2WI, T2-weighted image; MRI, magnetic resonance imaging.

had dropped to $7,991 \mathrm{mIU} / \mathrm{ml}$; however, all other blood test results remained normal. Enhanced pelvic magnetic resonance imaging (MRI) demonstrated an intramural mass that had partially invaded the myometrium and the posterior wall of the uterine fundus, and was isolated from the uterine cavity (Fig. 2). Since the patient's $\beta$-hCG levels had significantly decreased, they were kept under observation and had their $\beta$-hCG levels were monitored regularly. Serum progesterone was $2.3 \mathrm{ng} / \mathrm{ml}$.

One week later, a USG scan identified a heterogeneous mass measuring $4.9 \times 4.8 \mathrm{~cm}$ in the left wall of the uterine fundus reaching the serosa, with several anechoic areas inside the mass demonstrating significant circumferential vascularity. A preliminary diagnosis of choriocarcinoma was made; however, intramural pregnancy was not excluded. The decision to initiate chemotherapy was made in order to reduce the activity of trophoblastic cells, followed by exploratory surgery to confirm the diagnosis. A 5-day regimen of intramuscular methotrexate (MTX; $20 \mathrm{mg} / \mathrm{day}$ ) injections was prescribed. Following the 1st day of MTX treatment, the patient's serum $\beta$-hCG level had decreased to $3,893 \mathrm{mIU} / \mathrm{ml}$, and a USG scan revealed an increase in mass size $(5.6 \times 4.9 \mathrm{~cm})$ in the left uterine wall with substantial vascularity and tortuous vessels around the uterus. Three days following the initiation of chemotherapy, the patient exhibited fever, fatigue, mouth ulcers and loss of appetite; in addition, the patient's white blood cell count had decreased from $7.64 \times 10^{9}$ to $2.02 \times 10^{9}$ cells/1. On the 7 th day of chemotherapy, the patient's serum alanine transaminase level had increased from 35 to $71 \mathrm{U} / 1$ and their platelet count had decreased from $245 \times 10^{9}$ to $76 \times 10^{9}$ platelets $/ 1$.

Due to a minimal reduction in mass size and the evident side effects of chemotherapy, including severe bone marrow suppression, the decision was made to perform a hysteroscopy and exploratory laparotomy. No intrauterine pregnancy tissue was observed during the hysteroscopy and the left ostium of the fallopian tube was not easily delineated. Total blood loss did not exceed $10 \mathrm{ml}$. The laparotomy revealed a cystic projection measuring $4 \times 4 \mathrm{~cm}$ that was located on the left horn of uterus with a purplish-blue colored surface. In addition, the vascular vessels surrounding the left side of the uterus were convoluted with both adnexa considered normal (Fig. 3A and B). Following ligation of the uterine vessels, the lesion was completely removed. The lesion tissue was 
A

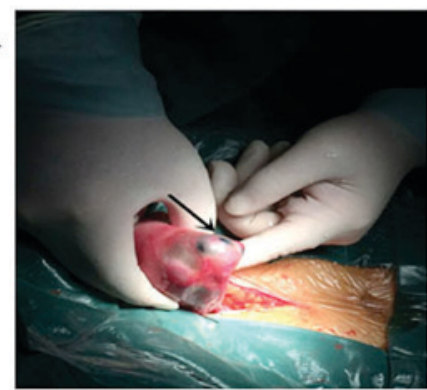

C

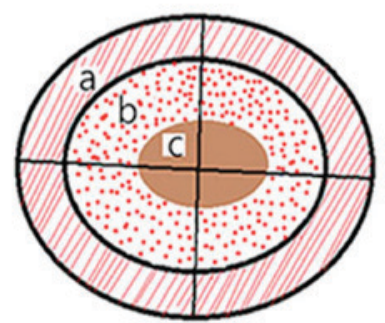

B

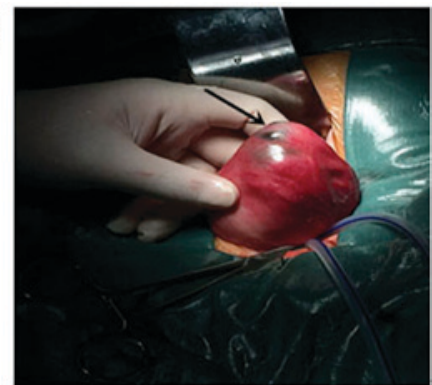

D

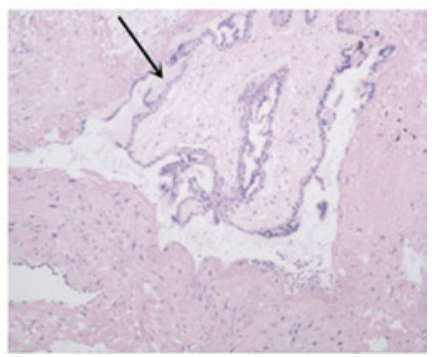

Figure 3. Lesion appearance, schema chart and pathological section. (A) Laparotomy revealed a mass inside the left posterior lateral aspect of the uterus. (B) Image of the mass removed. (C) Schema chart of the lesion section: a, normal muscle tissue in the outer layer; b, blood clot surrounding the vesicle; and c, edematous vesicle. (D) Pathological section demonstrating villi inside the uterine wall isolated from the uterine cavity, as demonstrated by the black arrow (hematoxylin and eosin stained; magnification, $\mathrm{x} 400$ ).

sectioned (thickness, $3 \mu \mathrm{m}$ ) and stained using hematoxylin and eosin prior to visualization using light microscopy. Pathological examination revealed a cystic lesion, centered with an edema vesicle-like structure of $\sim 1 \mathrm{~cm}$ in diameter, covered with honeycomb-like hemorrhages infiltrating the myometrium, with normal myometrium at the periphery (Fig. 3C). Placental villi identified in the myometrium supported a diagnosis of intramural pregnancy (Fig. 3D). Furthermore, the presence of ectopic endometrial tissue within the myometrium supported an additional diagnosis of adenomyosis. A total of 1 day following surgery, the patient's $\beta$-hCG level was $352.8 \mathrm{mIU} / \mathrm{ml}$ and 5 days post-operatively their $\beta$-hCG levels had decreased to $45.02 \mathrm{mIU} / \mathrm{ml}$, at which point the patient was discharged. A follow-up 1 week following discharge demonstrated that the patient's $\beta$-hCG level had returned to normal $(<30 \mathrm{mIU} / \mathrm{ml})$.

\section{Discussion}

In the case discussed in the present report, it was difficult to distinguish between intramural pregnancy and choriocarcinoma based on the clinical assessment. This is likely due to the rareness of intramural pregnancies experienced by medical professionals in clinical practice. A comprehensive text search on PubMed (National Center for Biotechnology Information, Bethesda MD, USA) using the query terms 'intramural ectopic pregnancy' returned 63 studies, of which the majority were case reports and frequently described interstitial pregnancy as intramural pregnancy. Intramural pregnancy should only include cases with elements of ectopic pregnancy within the myometrium, without any connection to the endometrial cavity or fallopian tubes. At present, there is no precise definition of intramural ectopic pregnancy. Intramural pregnancy should be treated as a specific type of ectopic pregnancy, similarly to interstitial pregnancy, caesarean scar pregnancy and cervical pregnancy, or should broadly include all of these conditions.
Currently, the etiology of intramural pregnancy is unclear and several hypotheses exist. The most accepted theory is that the embryo implants into the myometrium through a microscopic fistula, which may be the consequence of previous uterine surgery, such as a caesarean section or myomectomy (1). Similarly, the embryo may implant into the myometrium together with ectopic endometrial tissues during the development of adenomyosis (4). Another scenario is that the embryo may be implanted inside the myometrium artificially during IVF-ET (5). All the conditions mentioned above, except adenomyosis, were excluded in the present case report. There are several reports discussing the co-existence of adenomyosis with intramural pregnancy $(4,6)$. However, whether adenomyosis is the method of pathogenesis or serves as a high-risk factor for intramural pregnancy remains unclear.

Numerous studies have reported the early diagnosis of intramural pregnancy based on transvaginal USG and/or MRI (2,7-10). Memtsa et al (2) suggested several sets of criteria for the ultrasonographic diagnosis of intramural pregnancy and proposed a simple classification system that is useful in clinical practice. However, in the present case neither USG nor MRI could exclude the presence of choriocarcinoma. Furthermore, the results of the present case report suggest that the diagnosis of intramural pregnancy should be based on post-operative pathology results. The differential diagnosis between intramural pregnancy and GTD is clinically important due to their different treatment regimes. GTDs are a group of malignant disorders that require systemic chemotherapy. Choriocarcinoma should be considered if $\beta$-hCG levels continue to rise or do not decrease to an acceptable level post-partum or post-abortion. Metastasis of choriocarcinoma to other organs will induce corresponding symptoms, such as intracranial hemorrhage and hemoptysis (11). Although serum $\beta$-hCG levels may be increased in intramural pregnancy and choriocarcinoma, they are higher in choriocarcinoma compared with ectopic pregnancy, the latter of which rarely 
exceeds 10,000 mIU/l. Dousias et al (12) and Hsieh et al (13) reported two separate cases of intramural pregnancy with a negative maternal serum $\beta$-hCG. In the present case, the patient's previous menstrual cycles were regular with a recent history of amenorrhea for 40 days, which suggested a possible pregnancy. However, ultrasound examination revealed an empty uterine cavity. A high initial $\beta$-hCG concentration that declined (from 14,153 to 7,991 mIU/ml) excluded a diagnosis of tubal pregnancy and choriocarcinoma, respectively. Furthermore, the patient did not present with any typical ectopic pregnancy or miscarriage symptoms, such as abdominal pain or vaginal bleeding. USG and MRI scan results did not differentiate between GTD and intramural pregnancy.

The treatment for patients with intramural pregnancy should be individualized, depending on the location of the lesion, depth of muscular invasion, gestational age and the desire for future fertility (2). Reported successful treatment options for intramural pregnancy include the following: MTX-based chemotherapy, which can be delivered locally under ultrasound guidance (6), systemically (14) or injected directly into the embryo in combination with potassium chloride $^{(8)}$; laparoscopic surgical removal of lesions (15), although this option should be used with caution since the local blood flow is abundant and bleeding may be difficult to regulate; and uterine artery embolization (16). However, choriocarcinoma requires multiple cycles of chemotherapy compared with the MTX-based treatment for intramural pregnancy. As clinical and radiological results could not direct a final diagnosis in the current case, MTX-based chemotherapy was firstly chosen to reduce the activity of trophoblastic cells, as it is able to treat choriocarcinoma and intramural pregnancy. In the present case, unsatisfactory reductions in mass size and the side effects of chemotherapy resulted in the decision to perform a hysteroscopy and exploratory laparotomy. To prevent excessive blood loss during surgery, the ascending uterine artery was ligated, blocking blood flow to the bilateral infundibulopelvic ligament prior to excision of the lesion. In the majority of cases, conservative treatment of intramural pregnancy has poor efficacy and eventually leads to the need for surgery. The laparotomy performed confirmed a final diagnosis of intramural pregnancy with adenomyosis and treated the lesion effectively.

In conclusion, the present case report demonstrates that it is difficult to distinguish intramural pregnancy from choriocarcinoma. Under such circumstances, earlier surgical intervention would be an appropriate choice to diagnose and treat the disease simultaneously. In addition, surgical intervention reduces the potential toxic side effects of excessive chemotherapy due to a misdiagnosis of choriocarcinoma. Although intramural pregnancy is a rare condition, it is essential to establish a precise definition and classification system, in addition to diagnostic and treatment guidelines, for intramural pregnancy in order to effectively treat sufferers.

\section{Acknowledgements}

The present study was supported by the National Natural Science Foundation of China (grant no. 81172488) and the Outstanding Young Scientists Foundation of Shandong Province (grant no. BS2013YY035).

\section{References}

1. Lu HF, Sheu BC, Shih JC, Chang YL, Torng PL and Huang SC: Intramural ectopic pregnancy. Sonographic picture and its relation with adenomyosis. Acta Obstet Gynecol Scand 76: 886-889, 1997.

2. Memtsa M, Jamil A, Sebire N, Jauniaux E and Jurkovic D: Diagnosis and management of intramural ectopic pregnancy. Ultrasound Obstet Gynecol 42: 359-362, 2013.

3. Tulandi T (ed.): Ectopic Pregnancy. A Clinical Casebook. Springer International Publishing Switzerland, pp 115-122, 2015.

4. Karakök M, Balat O, Sari I, Kocer NE and Erdogan R: Early diagnosed intramural ectopic pregnancy associated with adenomyosis: Report of an unusual case. Clin Exp Obstet Gynecol 29: 217-218, 2002.

5. Khalifa Y, Redgment CJ, Yazdani N, Taranissi M and Craft IL: Intramural pregnancy following difficult embryo transfer. Hum Reprod 9: 2427-2428, 1994.

6. Choi DH, Kwon H, Kim YS and Kim JH: Intramural pregnancy associated with adenomyosis after in vitro fertilization and embryo transfer: A case report. J Reprod Med 54: 255-258, 2009.

7. Wang J and Xie X: Sonographic diagnosis of intramural pregnancy. J Ultrasound Med 32: 2215-2217, 2013.

8. Ko HS, Lee Y, Lee HJ, Park IY, Chung DY, Kim SP, Park TC and Shin JC: Sonographic and MR findings in 2 cases of intramural pregnancy treated conservatively. J Clin Ultrasound 34: 356-360, 2006.

9. Ong C, Su LL, Chia D, Choolani M and Biswas A: Sonographic diagnosis and successful medical management of an intramural ectopic pregnancy. J Clin Ultrasound 38: 320-324, 2010.

10. Katano K, Ikuta K, Matsubara H, Oya N, Nishio M and Suzumori K: A case of successful conservative chemotherapy for intramural pregnancy. Fertil Steril 72: 744-746, 1999.

11. Soper JT, Mutch DG and Schink JC; American College of Obstetricians and Gynecologists: Diagnosis and treatment of gestational trophoblastic disease: ACOG Practice Bulletin No. 53. Gynecol Oncol 93: 575-585, 2004.

12. Dousias V, Stefos T, Chouliara S, Stefanou D, Kamina S and Lolis D: Intramural pregnancy with negative maternal serum b-HCG. Eur J Obstet Gynecol Reprod Biol 111: 94-95, 2003.

13. Hsieh YY, Chang CC, Tsai HD, Yeh LS, Hsu TY and Yang TC: Intramural pregnancy with negative maternal serum beta-hCG. A case report. J Reprod Med 43: 468-470, 1998.

14. Ko HS, Lee Y, Lee HJ, Park IY, Chung DY, Kim SP, Park TC and Shin JC: Sonographic and MR Findings in 2 case of intramural pregnancy treated conservatively. J Clin Ultrasound 34: 356-360, 2006.

15. YeKuang, Chen XH, Si Y and Kong XC: Preoperative diagnosis and successful laparoscopic management of intramural pregnancy: Case report. Eur J Obstet Gynecol Reprod Biol 171: 385-386, 2013.

16. Zhuang Y and Huang L: Uterine artery embolization compared with methotrexate for the management of pregnancy implanted within a cesarean scar. Am J Obstet Gynecol 201: 152.e1-e3, 2009. 